\title{
Identification of a New Splice Variant of the Human ABCC6 Transporter
}

\section{Maria Francesca Armentano, ${ }^{1}$ Angela Ostuni, ${ }^{1}$ Vittoria Infantino, ${ }^{2}$ Vito Iacobazzi, ${ }^{2}$ Maria Antonietta Castiglione Morelli, ${ }^{1}$ and Faustino Bisaccia ${ }^{1}$}

\author{
${ }^{1}$ Department of Chemistry, University of Basilicata, 85100 Potenza, Italy \\ ${ }^{2}$ Department of Pharmaco Biology, University of Bari, 75100 Bari, Italy \\ Correspondence should be addressed to Faustino Bisaccia, faustino.bisaccia@unibas.it
}

Received 11 August 2008; Accepted 28 September 2008

Recommended by Anita H. Corbett

\begin{abstract}
ABCC6 is a member of the adenosine triphosphate-binding cassette (ABC) gene subfamily $\mathrm{C}$ that encodes a protein (MRP6) involved in active transport of intracellular compounds to the extracellular environment. Mutations in ABCC6 cause pseudoxanthoma elasticum (PXE), an autosomal recessive disorder of the connective tissue characterized by progressive calcification of elastic structures in the skin, the eyes, and the cardiovascular system. MRP6 is codified by 31 exons and contains 1503 amino acids. In addition to a full-length transcript of ABCC6, we have identified an alternatively spliced variant of ABCC6 from a cDNA of human liver that lacks exons 19 and 24. The novel isoform was named ABCC6 $\Delta 19 \Delta 24$. PCR analysis from cDNA of cell cultures of primary human hepatocites and embryonic kidney confirms the presence of the ABCC $6 \Delta 19 \Delta 24$ isoform. Western blot analysis of the embryonic kidney cells shows a band corresponding to the molecular weight of the truncated protein.

Copyright (c) 2008 Maria Francesca Armentano et al. This is an open access article distributed under the Creative Commons Attribution License, which permits unrestricted use, distribution, and reproduction in any medium, provided the original work is properly cited.
\end{abstract}

\section{Introduction}

ABCC6 belongs to the subfamily $\mathrm{C}$ of ATP-binding cassette $(\mathrm{ABC})$ transmembrane transporters. The ABCC6 gene consists of 31 exons encoding for a protein of 1503 amino acids and has 17 transmembrane spanning domains and two conserved intracellular nucleotide binding domains (NBDs). ABCC6 is homologous (45\% identity on amino acid level) to $A B C C 1$, known to confer multidrug resistance to tumor cells [1]; for that reason, ABCC6 was classified as a multidrug resistance associated protein and also named MRP6. The NBDs contain two highly conserved Walker motifs critical for ATP binding and transmembrane transporter functions [2]. Mutations of the ABCC6 gene cause the pseudoxanthoma elasticum (PXE) (OMIM 177850 and 264800), a multisystem disorder characterized by progressive calcification and degeneration of elastic fibers [3].

ABCC6 is highly expressed in human liver and to lesser extent in the proximal tubules of the kidney and only at very low levels, if at all, in tissues, such as skin, eyes, and cardiovascular system affected in pseudoxanthoma elasticum
(PXE) $[4,5]$. To date, genetic studies have identified 165 mutations, mainly missense and nonsense mutations, as well as large deletions (for a review see [6]). Since MRP6 is mainly expressed in liver and kidney, but only low levels are found in tissues affected by PXE, it has been suggested that PXE is primarily a metabolic disorder with secondary involvement of elastic fibers [7]. Despite the high correlation between ABCC6 mutations and PXE, the activity of MRP6 and its role in PXE remain largely unknown.

Recently, a splice variant leading to a $5 \mathrm{bp}$ deletion in the ABCC6 transcript has been associated with cardiac dystrophic calcifications in mice [8].

In our study, we report the identification of a new variant of ABCC6 from human liver cDNA lacking exons 19 and 24. This splice variant was also confirmed in hepatic and renal cell cultures.

\section{Materials and Methods}

Human liver and kidney BD Marathon-Ready cDNA were purchased from Clontech. Primary human hepatocites 
(Cambrex) were maintained in culture medium (Cambrex) following the manufacture's instructions. Human embryonic kidney cells (Sigma) were maintained in high glucose Dulbecco's modified Eagle's medium (DMEM) containing $10 \%(\mathrm{v} / \mathrm{v})$ fetal bovine serum, $2 \mathrm{mM}$ L-glutamine, $100 \mathrm{U}$ penicillin, and $100 \mu \mathrm{g} / \mathrm{mL}$ streptomycin at $37^{\circ} \mathrm{C}$ in $5 \% \mathrm{CO}_{2}$.

\subsection{Cloning of cDNA Encoding Human ABCC6}

To clone ABCC6 cDNA, the forward primer 5'-CACCATGGCCGCGCCTGCTG-3' and the reverse primer $5^{\prime}$-TCAGACCAGGCCTGACTCCTG-3' were designed to cloning the blunt-end PCR product into pcDNA 3.1D/V5-HisTOPO expression vector (Invitrogen). PCR was performed using human liver cDNA and Platinum PCR SuperMix (Invitrogen). The PCR was carried out on a PTC-100 Peltier Thermal Cycler (MJ Research) and it consisted of 1 cycle of $95^{\circ} \mathrm{C}$ for 2 minutes, 30 cycles of $94^{\circ} \mathrm{C}$ for 45 seconds, $62^{\circ} \mathrm{C}$ for 1 minute, $68^{\circ} \mathrm{C}$ for 5 minutes and 30 seconds, and $68^{\circ} \mathrm{C}$ for 10 minutes. PCR product was isolated from agarose gel, purified with the MinElute Gel Extraction kit (Qiagen) and ligated into pcDNA.3.1D/V5-His-TOPO expression vector. The recombinant vector was transformed into TOP10 E. coli cells. Individual clones were cultured overnight in Luria Bertani broth with $100 \mu \mathrm{g} / \mathrm{mL}$ ampicillin, and plasmid was isolated using the QIAprep Spin Miniprep kit (Qiagen).

\subsection{RT-PCR Analysis}

Total RNA was extracted from cultured cells using GenElute Mammalian Total RNA Miniprep Kit (Sigma). Before reverse transcription, the concentrations of total RNA were measured with the GeneQuant pro (Amersham International, Little Chalfont, UK) and RNA integrity was analyzed under UV light by visualization of 28S- and 18S-rRNA bands on a $1.5 \%$ agarose gel containing ethidium bromide. Total intact RNA $(1 \mu \mathrm{g})$ was reverse transcribed using GeneAmp RNA PCR Core Kit from Applied Biosystems with specific primers for the ABCC6 gene and MuLV reverse transcriptase, according to the manufacturers' instructions. Transcription reactions without the reverse transcriptase enzyme were performed for negative controls in subsequent PCR reactions.

To amplify region from exon 18 to exon 25 we used the following primers: $5^{\prime}$-GGCATGAATCTCTCCGGAG-3' (forward primer in exon 18) and $5^{\prime}$-CTGGAGGGCAGCAGAGAC-3' (reverse primer in exon 25). The PCR was performed on human liver and kidney cDNA and cDNA of cell cultures. PCR consisted of 1 cycle of $95^{\circ} \mathrm{C}$ for 2 minutes; 30 cycles of $94^{\circ} \mathrm{C}$ for 45 seconds, $58^{\circ} \mathrm{C}$ for 1 minute, $72^{\circ} \mathrm{C}$ for 2.5 minutes, and $72^{\circ} \mathrm{C}$ for 10 minutes. An aliquot of each amplicon was analyzed by ethidium bromide visualization on a $1.5 \%$ agarose gel to assess the size of the fragment, purified from the gel and directly sequenced.

\subsection{Sequencing}

The sequences of ABCC6 gene in the recombinant vector were verified using BigDye Terminator v3.1 Cycle Sequencing

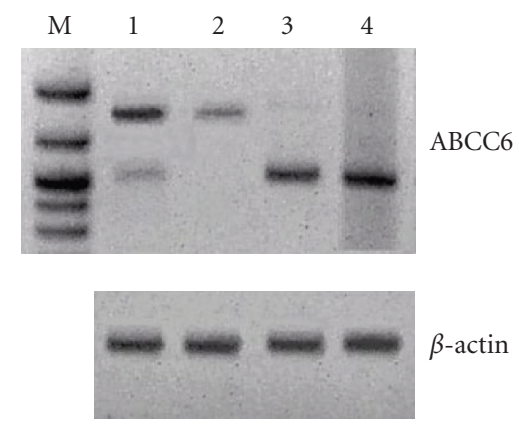

Figure 1: (Top) Expression pattern of exon18-exon25 region of ABCC6 from commercially available human liver and kidney cDNA (lanes 1 and 2), RNA reverse transcribed of primary human hepatocites (lane 3), and human embryonic kidney (lane 4). (Bottom) RT-PCR with $\beta$-actin primers as a control. The obtained PCR products were visualized by EtBr-stained agarose gel electrophoresis.

Kit (Applied Biosystems). The samples were analyzed with 3100 Avant Genetic Analyzer (Applied Biosystems) according to the manufacture's recommendation. The cDNA sequence of ABCC6 and its splicing variant ABCC6 $\Delta 19 \Delta 24$ have been deposited to GenBank under IDs AM774324 and AM711638, respectively. The sequences of PCR products obtained by the amplification of exon18-exon 25 region from cell cultures and from human liver and kidney cDNA have been performed by MWG Biotech.

\subsection{Western Blotting}

For immunoblot analysis of MRP6 expression, the whole cell lysate was isolated. HEK293 supplemented with protease inhibitors $(0.1 \mathrm{mM}$ PMSF, $5 \mu \mathrm{g} / \mathrm{mL}$ aprotinin, $5 \mu \mathrm{g} / \mathrm{mL}$ leupeptin, and $1 \mu \mathrm{g} / \mathrm{mL}$ pepstatin) were centrifuged at $1200 \mathrm{rpm}$ for 5 minutes. The pellet was resuspended in $1 \mathrm{~mL}$ of ice cold RIPA buffer (PBS, Igepal CA-630 1\%, sodium deoxycholate $0,5 \%$, SDS $0.1 \%$ ) and incubated on ice for 30 minutes; further disruption was obtained by sonication. After sonication, the lysate was incubated on ice for 30 minutes and then centrifuged at $10000 \mathrm{xg}$ for 10 minutes at $4^{\circ} \mathrm{C}$. The proteins were precipitated with five-volume of acetone at $-20^{\circ} \mathrm{Co} / \mathrm{n}$, resuspended in Laemmli buffer and separated by SDS-PAGE (7\%). Afterwards, the electrotransfer to an Immobilon-P transfer membrane (Millipore, Bedford, Mass., US) was confirmed by the reversible staining with Ponceau Red. After 20 minutes in incubation buffer (IB) $(50 \mathrm{mM}$ Tris, $150 \mathrm{mM}$ $\mathrm{NaCl}, 0,5 \%$ Tween-20), membrane was incubated for 1 hour with a polyclonal human antibody, raised against amminoacids 1-70 of human MRP6 (Santa Cruz Biotechnology, Inc.), diluted 1:400 in IB. After three washings with washing buffer (WB) (50mM Tris, $150 \mathrm{mM} \mathrm{NaCl}$ ), the membrane was incubated with an horseradish peroxidase conjugated goat antirabbit antibody (Sigma immunochemical, St. Louis, Mo., USA), diluted 1:5000 in IB. Finally, the blot was washed three times with $\mathrm{WB}$ and the proteins were visualized with ECL (Immun-Star HRP, Biorad, Hercules, Calif., USA). 


\section{Results and Discussion}

ABCC6 was amplified by PCR from human liver cDNA using specific primers and cloned into pCDNA3.1 vector. Surprisingly, the sequencing of some clones showed two different sequences of 4512 and 4137 nucleotides corresponding to the full length (ABCC6) and a shorter variant of ABCC6, respectively. A comparison of the shorter one with the exon/intron boundaries of ABCC6 gene revealed that the exons 19 and 24 were missing.

In order to verify if the short form, namely, ABCC6 $\Delta 19 \Delta 24$, was a result of low-frequency splicing events or an ABCC6 variant, we amplified and sequenced the PCR products of the exon 18-25 region from cDNA of human liver, human kidney, primary human hepatocites (HI), and human embryonic kidney cells (HEK293). Liver and kidney showed essentially the complete exon 18-25 region (Figure 1 lanes 1 and 2), whereas the variant in which both exons are missing has been found mainly in $\mathrm{HI}$ and HEK293 (Figure 1 lanes 3 and 4). Then, we suggest that the isoform lacking exons 19 and 24 may be a product of a splicing variant differently distributed in various tissues and cell lines.

Deletion of the entire exon 19 causes a shift of the reading frame with insertion of a stop signal at nucleotides 2614-2616 (Figure 2). As consequence of the premature stop codon, the putative novel protein has a different and shorter C-terminus than that of native MRP6 protein. By a secondround nested PCR, aberrant splicing of the ABCC6 mRNA has been previously observed in tissues that do not express appreciable amount of the protein [5]. More recently, it has been demonstrated that a missense mutation in ABCC6 gene of mice creates a premature stop codon that, apart from PXE, causes dystrophic cardiac calcification [8]. These findings suggest that truncated forms of ABCC6 can somehow affect cell activity.

For this reason, the identification of different splicing variants of ABCC6 in tissues such as liver and kidney, where ABCC6 is normally expressed, may be an important step in understanding the complex function of this gene and in clarifying the pathogenetic mechanisms of the correlated diseases.

To examine if the shorter variant encodes an expressed protein, we analyzed HEK293 by western blot analysis using an MRP6 N-terminus antibody as described in the methods (Figure 3). The predicted wild type protein of nearly $165 \mathrm{kDa}$ (MRP6) and a more intense band of about $100 \mathrm{kDa}$ corresponding to the truncated protein (MRP6 $\Delta 19 \Delta 24)$ are detected. The additional band between them could correspond to a different glycosylated form of the truncated protein. Western blot analysis is consistent with the expression level of the ABCC6 $\Delta 19 \Delta 24$ variant showed in Figure 1 and suggests that this isoform prevails in these cells.

Different speculative hypothesis on the function of the ABCC6 $\Delta 19 \Delta 24$ variant may be put forward.

The finding that ABCC $6 \Delta 19 \Delta 24$ variant codifies an expressed protein suggests a variety of functions for the

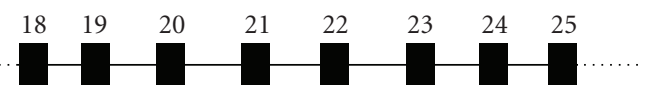

(a)

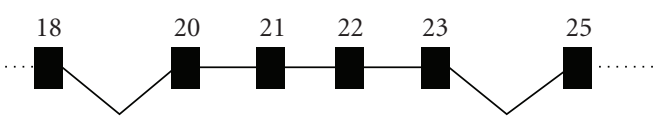

(b)

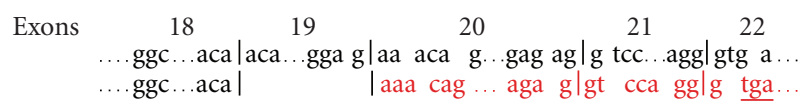

(c)

Figure 2: Splice event of the ABCC6 $\Delta 19 \Delta 24$. (a) ABCC6 wild transcript; (b) splice event of exons 19 and 24; (c) shift of open reading frame as a consequence of splicing of exon 19 is indicated in red characters; a premature stop codon in exon 22 is underlined.

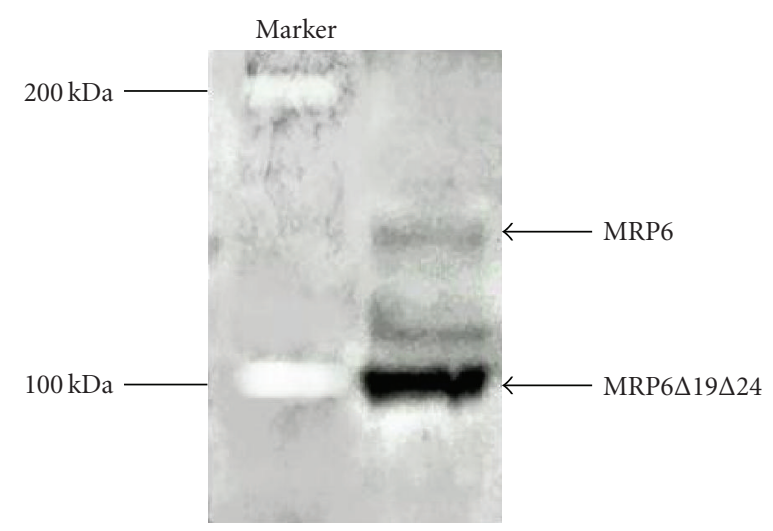

Figure 3: Western blot analysis of MRP6 in HEK293. The membrane probed with an MRP6 N-term specific antibody shows a clear band of about $100 \mathrm{kDa}$, corresponding to the translated protein from the ABCC6 $\Delta 19 \Delta 24$ isoform (MRP6 $\Delta 19 \Delta 24)$.

ABCC6 gene and confirms that the alternative splicing is a diffuse mechanism to increase protein diversity in the $A B C$ transporter superfamily.

We suggest that the translated protein of the ABCC6 $\triangle$ $19 \Delta 24$ variant is a half transporter. In fact, translation of the nucleotide sequence of this variant yields a putative truncated protein of 871 amino acids, encompassing the first two transmembrane domains and the first NBD at the C-terminal end. It is well known that some other human $\mathrm{ABC}$ genes encode half transporters as a consequence of alternative splicing, such as the ABCA5 gene, which encodes a protein of 1642 amminoacids and a polypeptide of 925 amminoacids [9], and the human ABCB6 that produces two distinct molecular weight forms, localized in the outer mitochondrial membrane and in the plasma membrane [10]. 


\section{Abbreviations}

\begin{tabular}{|c|c|}
\hline $\mathrm{ABC}:$ & Adenosine triphosphate-binding cassette \\
\hline PXE: & Pseudoxanthoma elasticum \\
\hline ABCC6 $\Delta 19 \Delta 24:$ & $\begin{array}{l}\text { Adenosine triphosphate-binding cassette } \\
\text { subfamily C member } 6 \text { lacking exons } 19 \\
\text { and } 24\end{array}$ \\
\hline NBD: & Nucleotide binding domain \\
\hline MRP: & Multidrug resistance associated protein \\
\hline HI: & Primary human hepatocites \\
\hline HEK293: & Human embryonic kidney cells \\
\hline rRNA: & Ribosomal RNA \\
\hline MuLV: & Murine Leukemia Virus \\
\hline EtBr: & Ethidium bromide. \\
\hline
\end{tabular}

\section{References}

[1] M. Kool, M. van der Linden, M. de Haas, F. Baas, and P. Borst, "Expression of human MRP6, a homologue of the multidrug resistance protein gene $M R P 1$, in tissues and cancer cells," Cancer Research, vol. 59, no. 1, pp. 175-182, 1999.

[2] E. Biemans-Oldehinkel, M. K. Doeven, and B. Poolman, "ABC transporter architecture and regulatory roles of accessory domains," FEBS Letters, vol. 580, no. 4, pp. 1023-1035, 2006.

[3] O. Le Saux, Z. Urban, C. Tschuch, et al., "Mutations in a gene encoding an $\mathrm{ABC}$ transporter cause pseudoxanthoma elasticum," Nature Genetics, vol. 25, no. 2, pp. 223-227, 2000.

[4] G. L. Scheffer, X. Hu, A. C. L. M. Pijnenborg, J. Wijnholds, A. A. B. Bergen, and R. J. Scheper, "MRP6 (ABCC6) detection in normal human tissues and tumors," Laboratory Investigation, vol. 82, no. 4, pp. 515-518, 2002.

[5] Y. Matsuzaki, A. Nakano, Q.-J. Jiang, L. Pulkkinen, and J. Uitto, "Tissue-specific expression of the ABCC6 gene," Journal of Investigative Dermatology, vol. 125, no. 5, pp. 900-905, 2005.

[6] A. S. Plomp, R. J. Florijn, J. ten Brink, et al., "ABCC6 mutations in pseudoxanthoma elasticum: an update including eight novel ones," Molecular Vision, vol. 14, pp. 118-124, 2008.

[7] J. Uitto, "Pseudoxanthoma elasticum-a connective tissue disease or a metabolic disorder at the genome/environment interface?" Journal of Investigative Dermatology, vol. 122, no. 3, pp. ix-x, 2004.

[8] Z. Aherrahrou, L. C. Doehring, E. M. Ehlers, et al., "An alternative splice variant in $A B C C 6$, the gene causing dystrophic calcification, leads to protein deficiency in $\mathrm{C} 3 \mathrm{H} / \mathrm{He}$ mice," The Journal of Biological Chemistry, vol. 283, no. 12, pp. 76087615, 2008.

[9] F. Petry, A. Kotthaus, and K. I. Hirsch-Ernst, "Cloning of human and rat ABCA5/Abca5 and detection of a human splice variant," Biochemical and Biophysical Research Communications, vol. 300, no. 2, pp. 343-350, 2003.

[10] J. K. Paterson, S. Shukla, C. M. Black, et al., "Human ABCB6 localizes to both the outer mitochondrial membrane and the plasma membrane," Biochemistry, vol. 46, no. 33, pp. 9443 9452, 2007. 

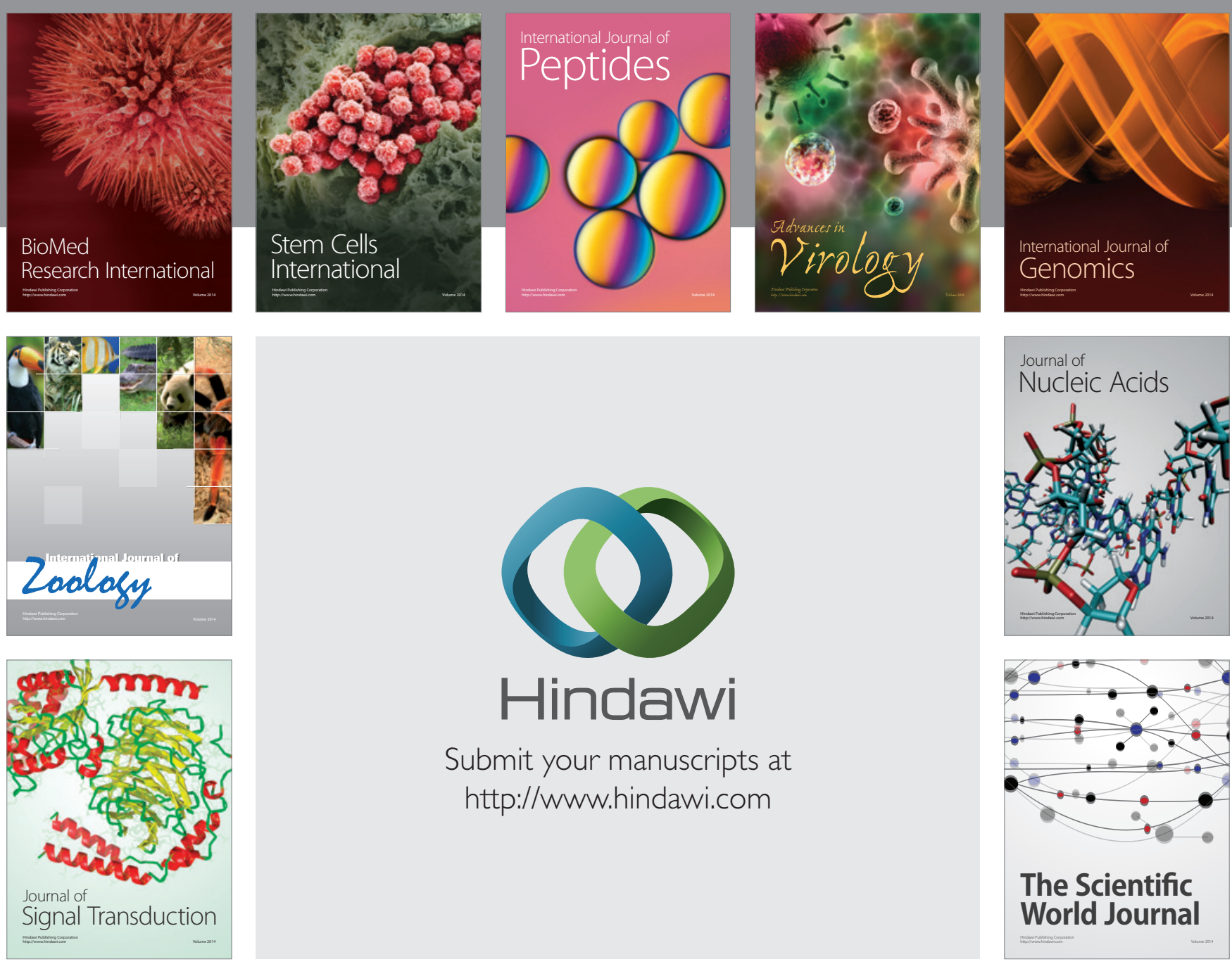

Submit your manuscripts at

http://www.hindawi.com
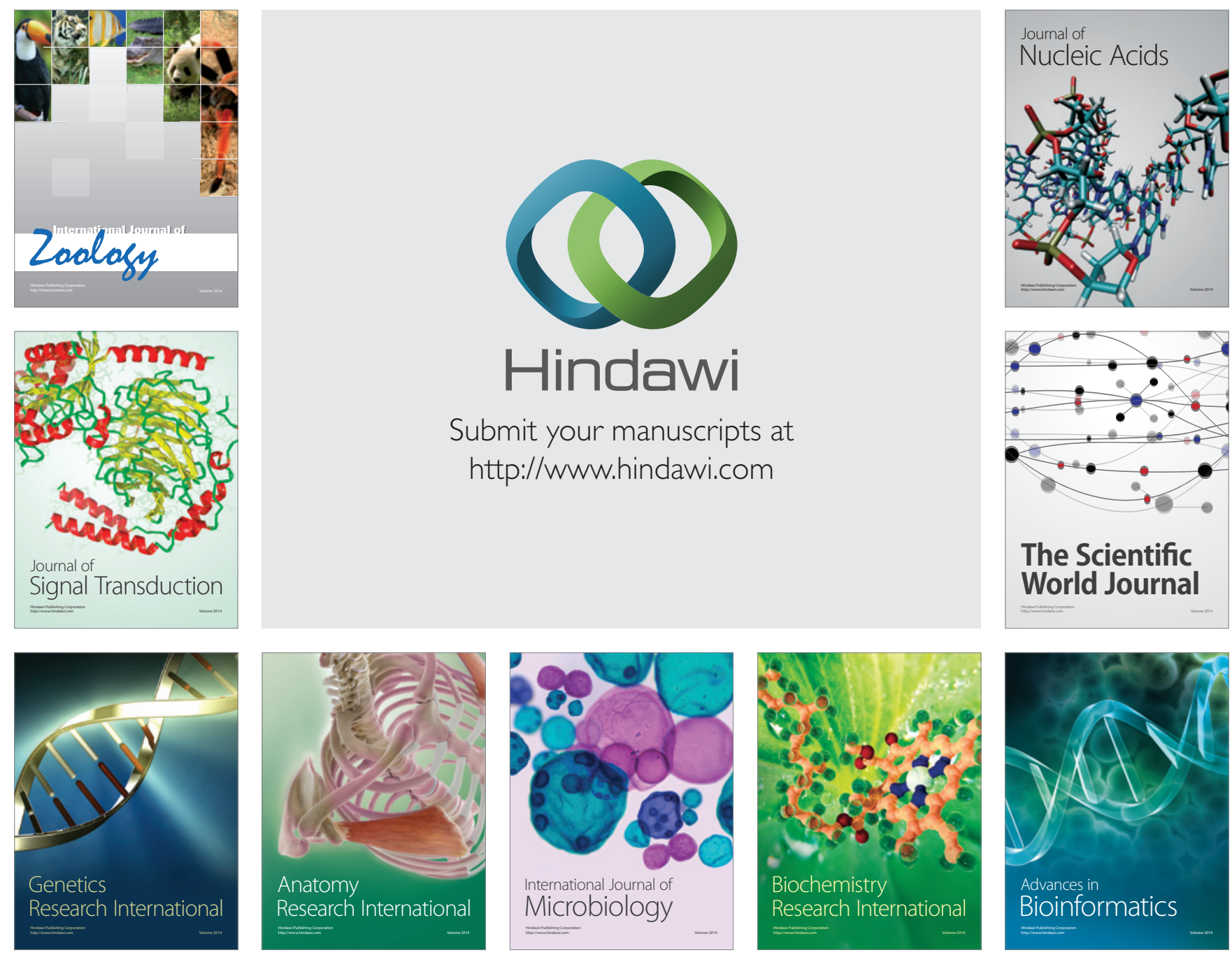

The Scientific World Journal
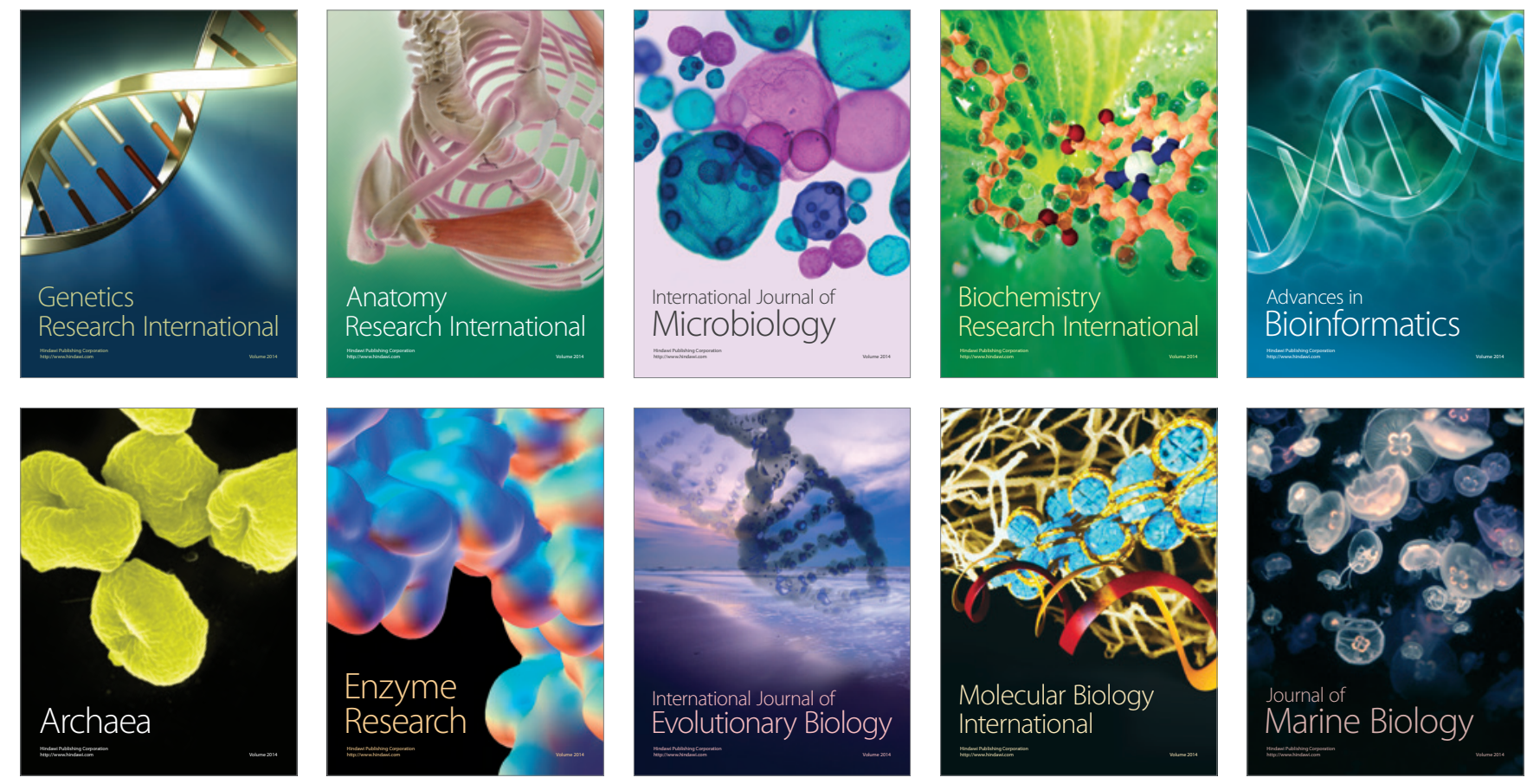